\title{
Corrigendum
}

\section{Corrigendum to "High Dose Dexmedetomidine: Effective as a Sole Agent Sedation for Children Undergoing MRI"}

\author{
Sheikh Sohail Ahmed, ${ }^{1}$ Tamara Unland, ${ }^{2}$ James E. Slaven, ${ }^{3}$ and Mara E. Nitu ${ }^{4}$ \\ ${ }^{1}$ Department of Pediatrics, Section of Pediatric Critical Care, Pediatric Sedation and Cardiovascular Intensive Care Unit, \\ Riley Hospital for Children at Indiana University Health, Indiana University School of Medicine, 705 Riley Hospital RI $49094 B$ \\ Indianapolis, IN 46202, USA \\ ${ }^{2}$ Department of Pediatrics, Pediatric Procedural Sedation, Riley Hospital for Children at IU Health North, 11700 N. Meridian Street, \\ Carmel, IN 46032, USA \\ ${ }^{3}$ Department of Biostatistics, Indiana University School of Medicine, 410 W. 10th Street, Suite 3000, Indianapolis, IN 46202, USA \\ ${ }^{4}$ Department of Pediatrics, Section of Pediatric Critical Care, Riley Hospital for Children at Indiana University Health, \\ Indiana University School of Medicine, 705 Riley Hospital Drive RI 4909 4B, Indianapolis, IN 46202, USA
}

Correspondence should be addressed to Sheikh Sohail Ahmed; ssahmed@iu.edu

Received 17 May 2015; Accepted 25 May 2015

Copyright (C) 2015 Sheikh Sohail Ahmed et al. This is an open access article distributed under the Creative Commons Attribution License, which permits unrestricted use, distribution, and reproduction in any medium, provided the original work is properly cited.

\begin{abstract}
A previous study conducted at the same institution, with a patient population of 77 , concluded that high dose dexmedetomidine can be successfully used for MRI sedation in children, with $29 \%$ of patients requiring additional agents for optimal sedation [19]. In our study, over the course of five years, the patient cohort was larger (544 patients) and included the previously reported 77-patient cohort. This larger patient cohort analysis validated previously reported data: $100 \%$ success of the existing sedation protocol, rounded up from $99.8 \%$ acknowledging one patient failure, with the adjunctive sedative agents used on $21.5 \%$ of cases.
\end{abstract}




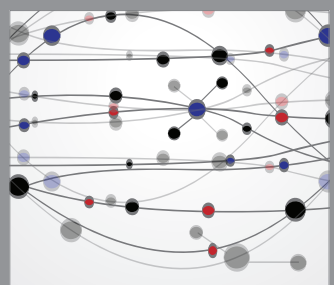

The Scientific World Journal
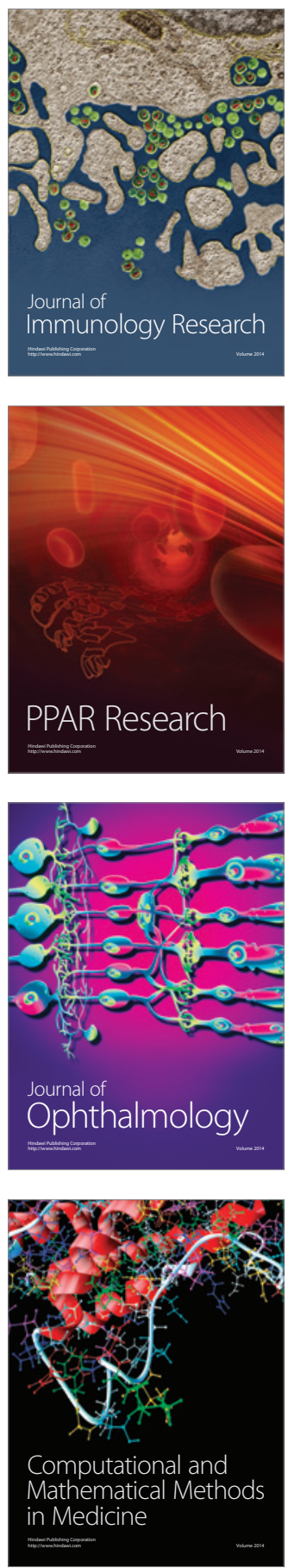

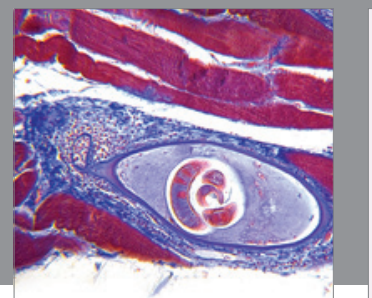

Gastroenterology

Research and Practice
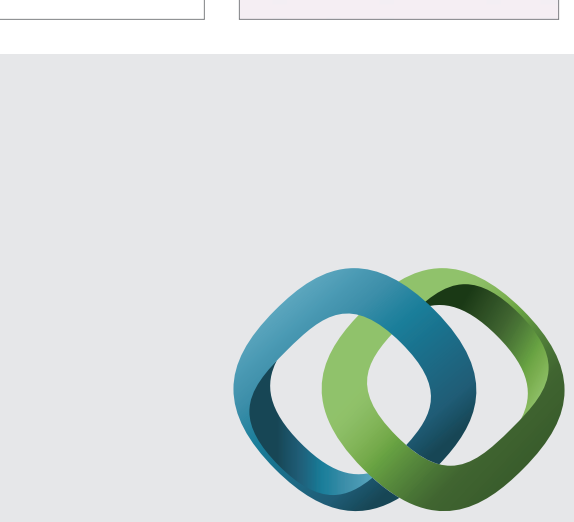

\section{Hindawi}

Submit your manuscripts at

http://www.hindawi.com
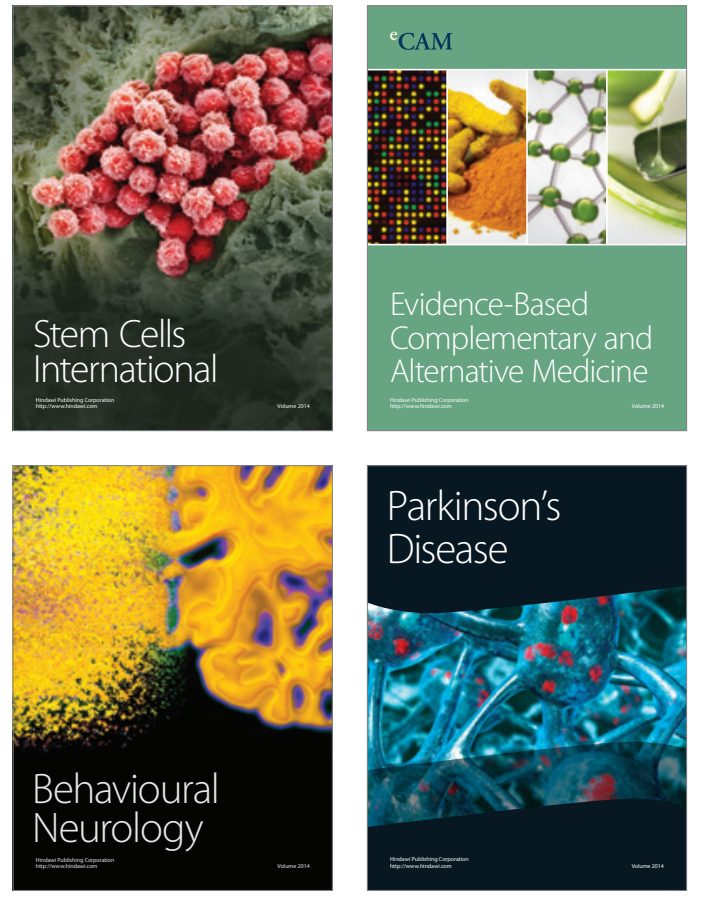
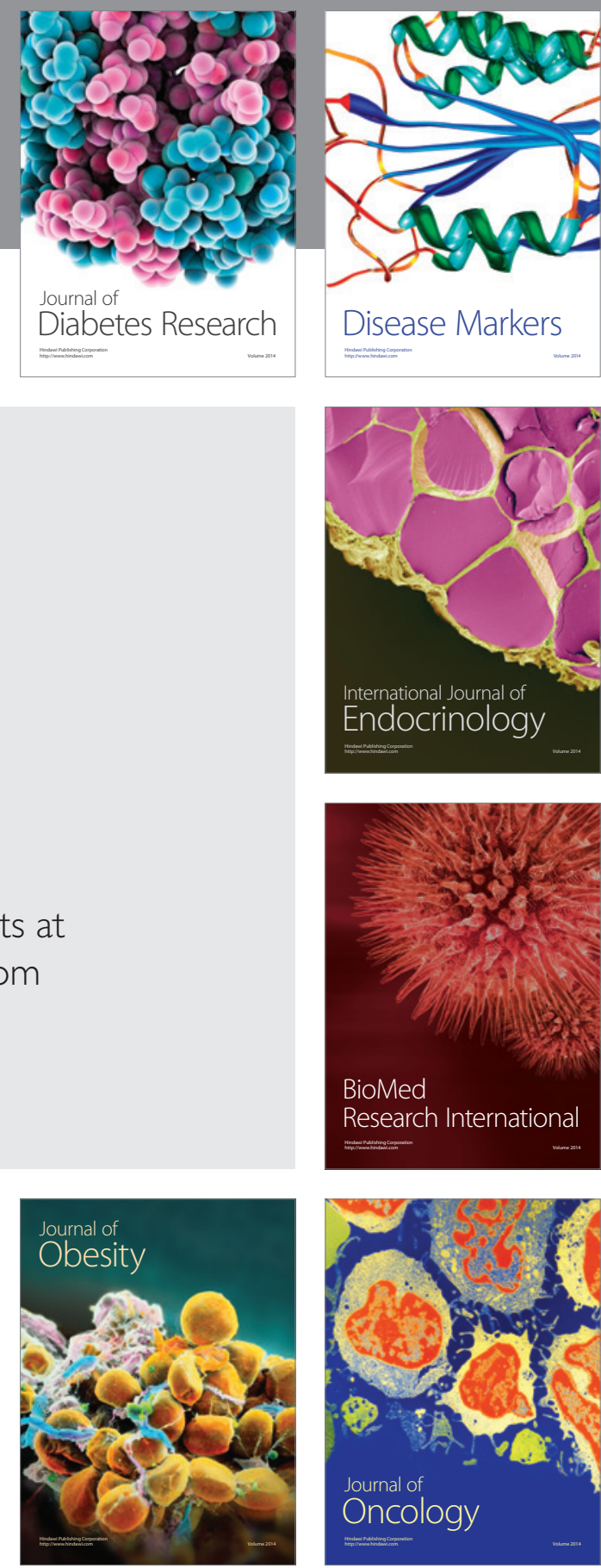

Disease Markers
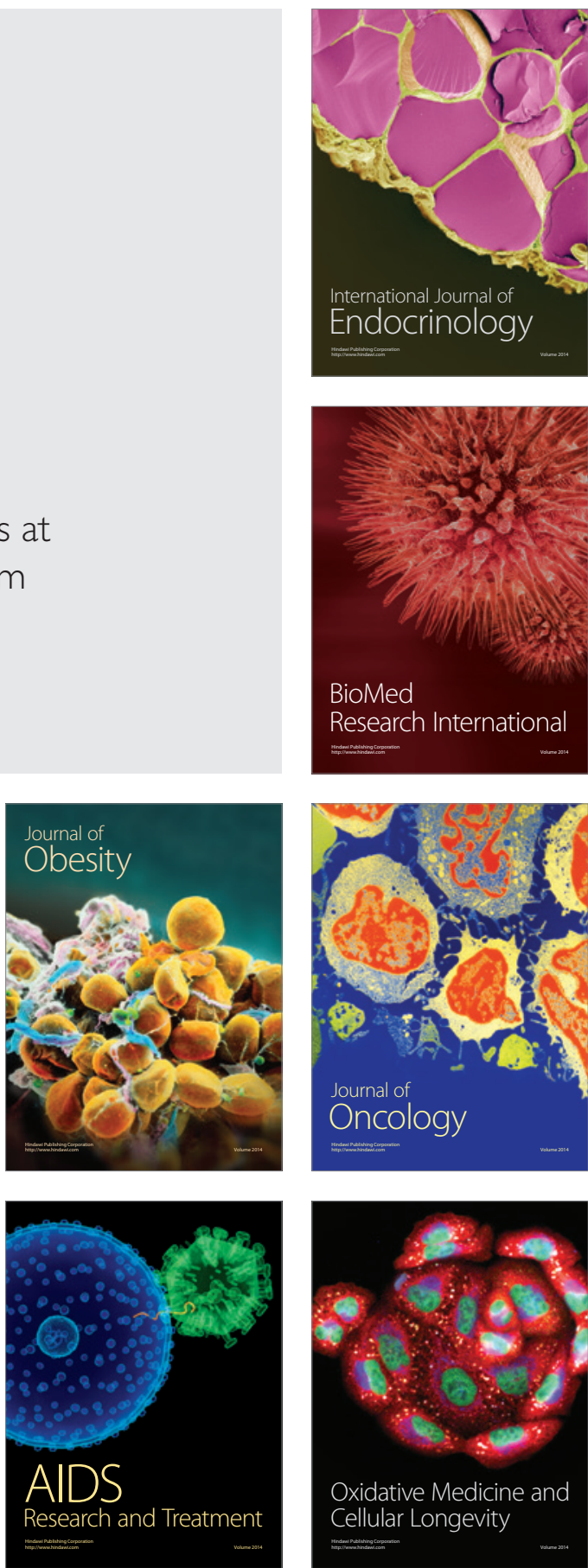